\title{
Electrochemistry supported by zeolites, clays, layered double hydroxides, ordered mesoporous (organo)silicas, and related materials
}

\author{
Antonio Doménech-Carbó $^{1}$ - Alain Walcarius ${ }^{2}$
}

Published online: 19 June 2015

(C) Springer-Verlag Berlin Heidelberg 2015

In the last decades, there is an increasingly growing interest in the electrochemistry of a series of ordered solids based on zeolites, clays, layered double hydroxides, and other materials having in common a nanostructured porous inorganic matrix able to be permeated with organic and inorganic species. Such materials comprise a variety of nanoarchitectures in relation to the shape and size of the crystals and their cavities, pores, channels, etc., from microporous to mesoporous, and a variety of guest species, from neutral molecules to cationic and anionic guests. The synergistic combination of site isolation and ion exchange or adsorption capacity of these materials has motivated their exploitation in the electrochemistry domain for catalysis, energy production and storage, and sensing. The current issue of Journal of Solid State Electrochemistry is devoted to present a picture of the state of the art in this field covering the different aspects directly dealing with the intersection between materials science and electrochemistry.

The issue presents an overview of various aspects of this electrochemistry: the existing theoretical approaches to the same are reviewed by A. Doménech-Carbó, emphasizing the interest in extending available models on the electrochemistry of ion-insertion solids to the study of porous materials. An important aspect to be considered is the electrosynthesis of such materials. Here, the electrochemically assisted synthesis of ordered mesoporous silicas and layered double hydroxides

Antonio Doménech-Carbó

antonio.domenech@uv.es

1 Department of Analytical Chemistry, University of Valencia, Burjassot, Valencia, Spain

2 Laboratoire de Chimie Physique et Microbiologie pour l'Environnement, UMR 7564 CNRS-Université de Lorraine, Villers-lès-Nancy, France is reviewed by A. Walcarius and C. Mousty, underlying the flexibility and performance of the electrosynthetic strategies. Chemical power sources based on layered double hydroxides, reviewed by W. Yang et al., are representative of the capabilities of these materials for energy generation. The scope of their electroanalytical applications can be seen in the review of organoclay-based electroanalysis from T. Kenfack et al. A comprehensive view of the electrochemistry of layered double hydroxides containing 3D metal cations is provided in the feature article of C. Mousty et al.

The issue covers different frontier contributions. The electrochemical control of ion exchange is illustrated by the study on proton exchange with sodium zeolites $\mathrm{X}$ and A presented by R. Dryfe et al., where it underlined the capability of electrochemical-assisted ion exchange for maintaining the structural integrity of the materials in contrast with conventional chemical methods.

The electrocatalytic performance of the different families of materials is one of the principal aspects to be treated for synthesis and electroanalysis. The catalysis of the oxygen reduction reaction, an important electrochemical process, using Pd-modified zeolite 13X is studied by Mojovic et al., while R. Li et al. present a study of the electrocatalytic oxidation of methanol on NiZnsalenA-modified glassy carbon electrode.

The use of electrochemistry to study the sorption properties of organo-montmorillonites is described by Z. Navratilova et al., whereas del Hierro et al. study the voltammetric characterization of titanium and zinc hybrid mesoporous SBA-15 materials with particular attention to the avoidance of leaching of ions. These works are illustrative of the possibility of using voltammetric measurements for obtaining relevant physicochemical information on this kind of materials.

The analytical applications of electrodes modified with the studied porous materials, often forming different nanocomposite systems, are illustrated by the articles of Y. Wen et al. 
on the determination of two trace phytohormones, H. ElDesoky et al. on the determination of pipazethate hydrochloride in blood, E.V. Benvenutti et al. on dopamine determination at mesoporous silica xerogel modified with bridged ionic silsesquioxane incorporating copper tetrasulfonated phthalocyanine, V. Ganesan et al. on the determination of nitrite with silver nanoparticle-anchored amine functionalized mesoporous silica, and I. Sierra et al. on the determination of lead in drinking waters using a bifunctional periodic mesoporous organosilica.

The research on the application of the considered materials in energy generation devices is illustrated by the contribution from V. Baglio et al. studying the influence on the electrochemistry of direct methanol fuel cells of the transport of methanol and protons in layered double hydroxide and smectite clay-based composites.

Finally, the aforementioned possibility of efficient electrosynthetic methodologies is exemplified by the contributions from C. Despas et al. describing the electrochemically assisted self-assembly of ordered and oriented mesoporous silica thin films bearing octyl or hexadecyl groups and J.A. Cox et al. on the surface immobilization of nanobeads allowing for the control of size, shape and distribution of pores in electrochemically generated sol-gel films.

In summary, this monographic issue presents a quite comprehensive view of the electrochemistry of micro- and mesoporous silica and aluminosilicate-based materials and layered double hydroxides where the reader can find representative examples of the theoretical and practical aspects, including synthesis, analysis, physical chemistry and energy management, which are a matter of research in the field of electrochemistry.

The guest editors thank to all the authors and reviewers that have contributed to this monographic issue as well as to Prof. Fritz Scholz, Editor-in-Chief of Journal of Solid State Electrochemistry, for his kind invitation and to Dr. Michael Hermes, Associate Editor, for his valuable technical assistance. 